\title{
A new method to improve the resolution of reconstructed heavy nuclei spectrum
}

\author{
L.T. Zhao ${ }^{* * 1}$, L.L.Ma ${ }^{2}$, S.S Zhang ${ }^{2}$, X.H.Chen ${ }^{1}$ \\ ${ }^{1}$ Institute of Physics, Liaoning University, ShenYang, China \\ ${ }^{2}$ Institute of High Energy Physics, CAS, BeiJing, China \\ E-mail: zhaolteihep.ac.cn
}

The accurate measurement of the spectra of elemental composition of cosmic rays is crucial to the origin of cosmic rays. The measurements of high energy cosmic rays are based on the ground experiments, and the composition reconstruction depends on the hadronic interaction model, so large uncertainties are introduced in the measurements. In this paper, we proposed a new method which is based on the characteristics of direct Cherenkov Light (DC-light) and LHAASO-WFCTA to improve resolution of reconstructed heavy nuclei spectrum. The method is independent of the interaction models for the heavy nuclei spectrum measurements.

\footnotetext{
*Speaker.
}

${ }^{\dagger}$ first author 


\section{Introduction}

After more than 100 years from the first observations of cosmic rays by Austrian scientist Hess, the origin, the acceleration and the propagation of cosmic rays still remains a central unresolved problem in astrophysics, voted one of the eight mystery of astronomy by scientific journals in 2012. Baade and Zwicky have proposed an idea in the paper that published in the 1934 [1], the cosmic rays come after supernova explosions. This idea has been become a main current in the astrophysics. For two reasons: firstly, the energy density of cosmic rays is exactly right to the supernova explosion effects [2], secondly, Fermi acceleration by supernova diffusive shock has good effects to the acceleration of cosmic rays . However, the majority of components for cosmic rays is proton, there is no strong evidence for supernova explosion to accelerate the proton. Additionally, measurements of energy spectrum for individual elements are very important to resolve the questions of the origin and the acceleration of cosmic rays. The measurement of cosmic rays includes two forms, one is direct measurements. Cosmic rays with energies lower than $100 \mathrm{TeV}$ can be detected by space experiment directly. Due to limited collection area of space experiments $\left(\sim 1 \mathrm{~m}^{2}\right)$ and decreasing flux of cosmic rays, it is difficult to take the effective measurements for events with energy higher than $100 \mathrm{TeV}$. The other one is indirect measurements, ground-based arrays can measure the cosmic rays beyond the few $\mathrm{TeV}$ by measuring extensive air showers (EAS) which is produced by the high energy cosmic rays interacting with nuclei in the atmosphere [3].However, the indirect measurements are dependent highly on the hadronic interaction models.

The direct Cherenkov light(DC-light) is produced by the primary incoming nucleus prior to its first interaction with the atmosphere. So it is independent of hadronic interaction models. The intensity of it is proportional to square of the charge of primary particle and sensitive to the primary compositions of cosmic rays [4]. In addition, due to the thin air at the top of the atmosphere, the emission angle of DC-light is in a narrow angle range $\left(0.1^{\circ} \sim 0.3^{\circ}\right)$. So the DC-light can be detected by the Cherenkov telescopes which can detect the photons with highly direction resolution.

In this paper, we describe the characteristics of DC-light in the Sec2, including the angle characteristic and distribution characteristics in the observation plane. In the Sec3, we introduce a new parameter to discriminate the iron events from proton events via the angle characteristics of DC-light, analyze the improvement of selection efficiency of the multi-variable analysis(MVA)by add the new parameter to Hillas selection parameters [5]. Further discussions and conclusions are given in section of Summary and Discussions.

\section{Characteristics of DC-light}

The Cherenkov light produced by the high energy cosmic rays is consisting of DC-light and EAS-light.The DC-light is emitted by primary cosmic rays before interacting with atmosphere at typically height of $30 \mathrm{~km}$, a light cone with a radius of roughly $100 \mathrm{~m}$ is formed on the ground. Compared with DC-light, the EAS-light is emitted by the secondary particles,a much wider and brighter light cone is formed on the ground. The EAS-light is the dominate background for the detection of DC-light. Thus, to discern the DC-light against the EAS-light, some distinguishing characteristics must be identified. Monte Carlo simulation is used to study the characteristics of DC-light in EAS. Cosmic rays showers are generated use CORSIKA [6]. Iron $(\mathrm{Z}=26)$ and proton 
$(\mathrm{Z}=1)$ are chosen as target particle. The primary zenith angles of protons and irons are sampled $0^{\circ}$. The height of observation plane with 4400m and QJSJETII-04 model for high energy hadronic process are chosen in the CORSIKA program. Lateral density distribution of DC-light and EASlight emitted by the iron and proton with energy of $50 \mathrm{TeV}, 100 \mathrm{TeV}, 500 \mathrm{TeV}$ on the observation plane are shown in Figure1. EAS-light intensity increases approximately linearly with the primary energy of cosmic rays. In order to emit the DC-light, primary energy of primary cosmic rays must exceed the Cherenkov threshold. If the primary energy of cosmic rays is much higher than the Cherenkov threshold, the amount of emitted DC-light is basically constant as shown in Figure 1, so there should be an optimal energy range to discern the DC-light against the EAS-light. Due to the radiation mechanism of DC-light and EAS-light, the ratio of density of DC-light to EASlight is increase with Rm (impact distance to the shower axis from the position of photons on the observation plane) and decrease with energy. The intensity of the DC-light is proportional to square of the charge of primary particle, the fact is checked by Figure 1. The DC-light intensity of proton is approximation to 0 , however, the DC-light intensity of iron increase with $\mathrm{Rm}$, reaching the maximum values $300 \mathrm{pe} / \mathrm{m}^{2}$. This characteristic provides a method to reconstruct the charge $\mathrm{Z}$ of primary cosmic rays to discriminate heavy nuclei from other components of the cosmic rays. The curve of DC-light density has a slightly fluctuation in the Rm range of $90 \mathrm{~m} \sim 110 \mathrm{~m}$, where approach the height of first interaction, even nuclei with the same first interaction depth suffer from large fluctuations, which due mainly to the details on how the first interaction proceeds, if the initial interaction yields a large number of low $\mathrm{Z}$ fragments, the DC-light yield is low. If the interaction yields only 1 or 2 low $\mathrm{Z}$ fragments and the original primary only loses a small amount of charge, then the DC-light yield is high, the different fragmentation process case there are large fluctuations

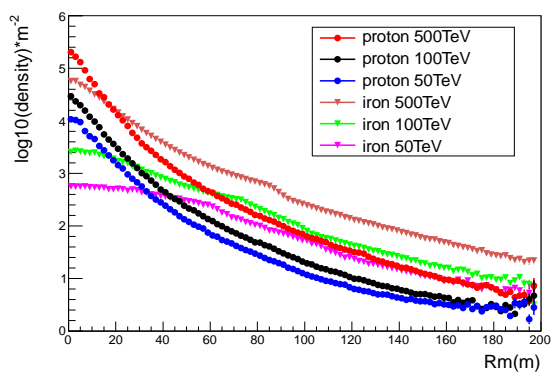

(a)

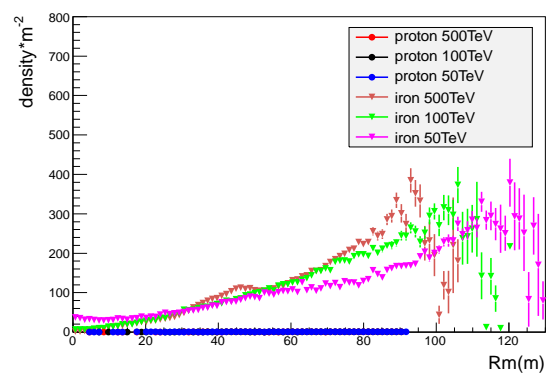

(b)

Figure 1: (a) Lateral density distribution of EAS-light emitted by the iron and proton. (b) Lateral density distribution of DC-light emitted by the iron and proton. The emission angle range of DC-light and EAS-light is chosen lesser than $0.25^{\circ}$, the reason is that the pixel of LHAASO-WFCTA angular resolution is $0.5^{\circ}$.

Because the DC-light is emitted at the top of the atmosphere, the emission angle of it is smaller than the emission angle of EAS-light. The typical emission angle for DC-light is from $0.1^{\circ}$ to $0.3^{\circ}$. However, the emission angle of most of EAS-light is greater than $0.4^{\circ}$, as shown in Figure 2. The significantly difference of the two plots is the Figure 2(a) has filamentary structure under the main structure, which is contributed by DC-light. So the DC-light should be imaged closer to the shower direction in the Cherenkov telescope focal plane, and can be measured by the IACTs with optimum pixel resolution. 


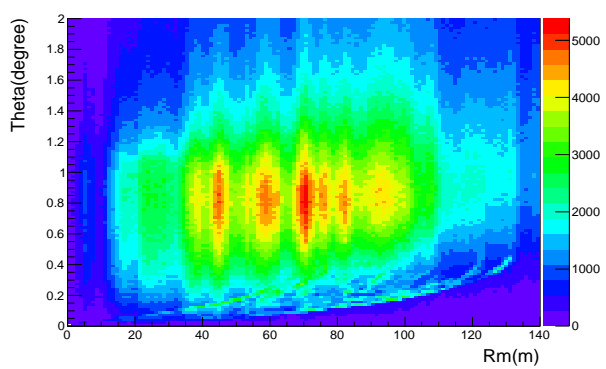

(a)

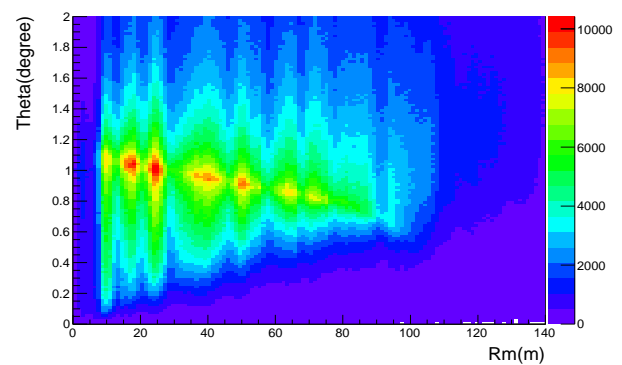

(b)

Figure 2: Simulated of emitted angle of DC-light and EAS-light relative to parameter Rm, (a) $50 \mathrm{TeV} \mathrm{Z}=$ 26 nuclei (iron), (b) $50 \mathrm{TeV} \mathrm{Z}=1$ nucleus (proton).

\section{Simulation and Analysis}

\subsection{Introductions of WFCTA and Simulation}

The project of LHAASO will be built in the haizi Mountain, Sichuan province, China, with an altitude of $4410 \mathrm{~m}$. It is mainly composed of :(1) $1 \mathrm{~km}^{2}$ array (KM2A), including 5195 electromagnetic detectors, and 1171 muon detectors, (2) $78000 \mathrm{~m}^{2}$ Water Cherenkov Detector Array(WCDA), (3)16 Wide Field of View Cherenkov Telescope Array (WFCTA) detectors. The hybrid observatory can be obtained by the three type detector arrays. The shower core positions and the arriving directions of the showers can be reconstructed by WCDA or KM2A. The energy of the showers can be reconstructed by WFCTA. The telescope of WFCTA is mainly composed of a camera and a $5.29 \mathrm{~m}^{2}$ spherical aluminized mirror, with a radius of curvature of $5800 \mathrm{~mm}$. The camera is placed at the focal plane which is $2870 \mathrm{~mm}$ away from the reflector center to optimize the spot shape.Each camera consists of an array of 1024 SiPMs, the 1024 SiPMs are followed the array of $32 \times 32$, the angular resolution of each SiPM is $0.5^{\circ}$. In order to increase the light collection efficiency, a Winston Cone is used in front of the SiPM.

In order to investigate the ability of WFCTA to discriminate the compositions of cosmic rays by using the DC-light, a simulation is done. The first step of the simulation is to generate showers with CORSIKA. The same number of protons and irons with energies between $30 \mathrm{TeV}$ to $500 \mathrm{TeV}$ are generated according to a power law spectrum with a spectral index of -2 . The events number is normalized to the expectation of Hördanel model [7]. In order to compare the effect of interaction models,two models QGSJETII -04 and EPOS LHC are selected. The primary zenith angle of nuclei are sampled from $18^{\circ}$ to $38^{\circ}$. Considering the field of view, the pointing direction of the telescopes in the zenith direction is set to $28^{\circ}$. The shower cores are sampled in a square of $300 \mathrm{~m} \times 300 \mathrm{~m}$. The second step of the simulation is the telescope simulation. The process of Cherenkov photons in the telescope is traced by a ray tracing procedure, the location and arrival time of the Cherenkov photons on the focus plane of WFCTA are obtained.The roughness of the mirrors surface is simulated by changing the directions of the normal randomly in a cone $0.2^{\circ}$. To simplify the progress of the simulation, the fast simulation mode is chosen. The SiPM will be triggered if it gets together the arriving photoelectrons more than 20pe, the number of trigger SiPMs is outnumber the 3 and gets together on the focal plane will trigger the WFCTA. 


\subsection{Selection Iron Events by The DC-light}

According to the angle characteristics of DC-light shown in the Figure 2, a parameter is defined to discriminate the iron events from other cosmic rays as shown in equation 3.1.

$$
\text { Ratio }=\frac{I_{\text {dir }}}{I_{\operatorname{cog}}}
$$

Where $I_{d i r}$ represents Cherenkov photon intensity of the pixel pointing to the arriving direction

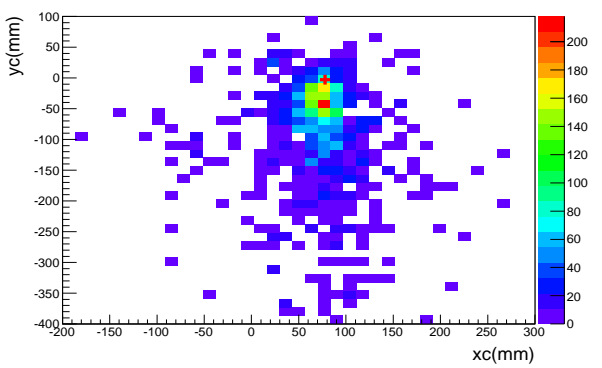

(a)

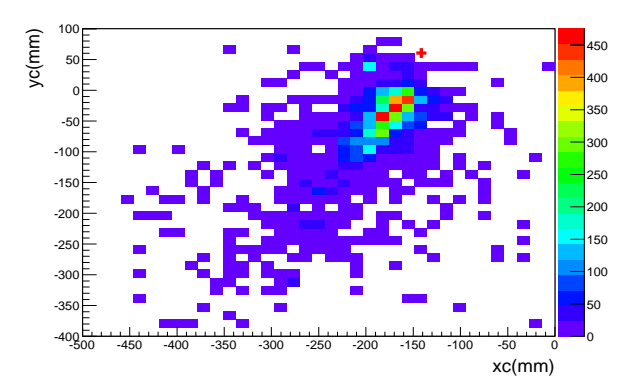

(c)

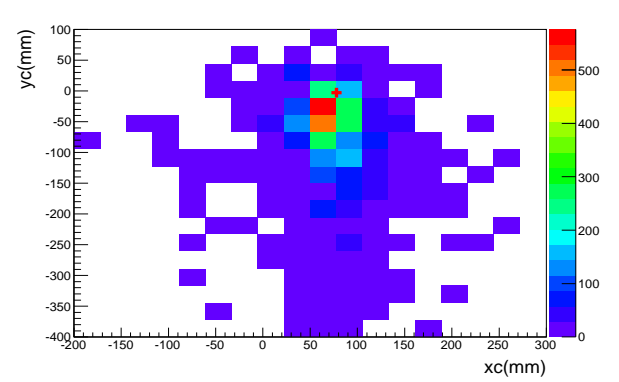

(b)

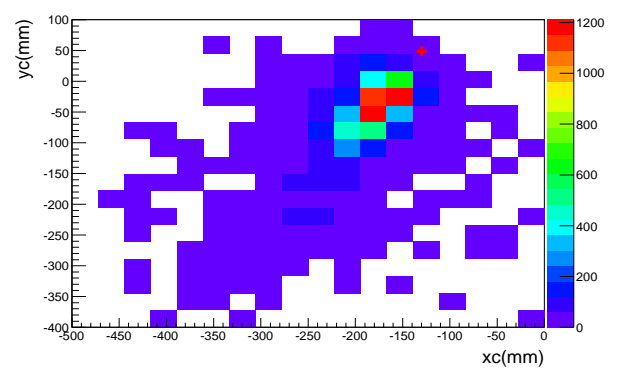

(d)

Figure 3: The image of Cherenkov photons on the difference resolution of pixel array, the Rp (which is defined as the impact distance to the shower axis from the telescope) is nearby $60 \mathrm{~m}$,the energy is $90 \mathrm{TeV}$. (a) Image of iron on the telescopes with pixel array resolution $0.25^{\circ}$. (b) Image of iron on the telescopes with pixel array resolution $0.5^{\circ}$. (c) Image of proton on the telescopes with pixel array resolution $0.25^{\circ}$. (d) Image of proton on the telescopes with pixel array resolution $0.5^{\circ}$. The reconstructed shower direction is shown by a red cross in each image.

(the direction pix $\triangle_{d i r}$ ) of showers, $I_{c o g}$ is Cherenkov photon intensity of pixel containing center of gravity (center pix $\triangle_{c o g}$ ) of the image. In order to study the ability of selection by the Ratio for the difference resolution of pixel array of telescopes,satisfy the requirements of optical design of WFCTA, a new array with $64 \times 64$ and the pixel resolution $0.25^{\circ}$ are simulated in our simulation process. Figure 3 shows the image of Cherenkov photons on the different resolution of pixel array. Because the DC-light images nearby $\triangle_{d i r}$, the intensity of $\triangle_{d i r}$ of iron is brighter than that of proton. The angular distance between the DC-light and the arriving direction of shower is about $0.1^{\circ} \sim 0.3^{\circ}$. Therefore, the difficulty of selecting the DC-light pixel against the much larger background of Cherenkov light that use the parameter of Ratio is decrease with the pixel resolution, because secondary particles produced at lower altitude are scattered, producing Cerenkov light which enters into the angular bin of the DC-light. 


\subsection{Improvement Selection Efficiency by the DC-light}

For accurate analysis the improvement of selection efficiency of the MVA by add Ratio to Hillas selection parameters, a set of Hillas parameters including the Size, Length, Width, and Dist, are calculated [5]. The Size is the total number of photoelectrons in shower image, Length and Width are the second moments of the light distribution along the major and minor axes of the image, the Dist is the angular distance between shower's arriving direction and center of gravity (COG) of the image. Before the process of the analysis, some events selecting criteria are used :(1) only COG from camera center is less than $6^{\circ}$ are used, (2) impact parameter Rp is chosen from $50 \mathrm{~m}$ to $170 \mathrm{~m},(3)$ to discard the events which have large fluctuations ,the ratio (Length/Width) should be less than 7, the angular distance of Dist should be less than $3.8^{\circ}$. The criteria is defined "selecting cut". From the process of the shower development, the $X_{\max }$ of iron should be about $70 \mathrm{~g} / \mathrm{cm}^{2}$ higher than that of proton for events with the same energies. The COG of image can be considered as the projection of Xmax on the camera plane, the Dist is related with the value of $X_{\max }$. Additionally, for a given energy and $\mathrm{Rp}$, Length/Width is a parameter sensitive to the depth of the shower maximum, that is related to the nature of the primary nuclei, so the parameters of Dist and Length/Width also can be used to discriminate compositions. Due to the multi-type detector arrays of LHAASO, the hybrid observation of air shower can be achieved. At the energy range from $30 \mathrm{TeV}$ to $500 \mathrm{TeV}$, the geometry reconstruction of the shower including the shower core reconstruction and arriving direction reconstruction can be achieved by WCDA, the resolution of which can be achieved $5 \mathrm{~m}$ and $0.1^{\circ}$, respectively, so the resolution of the reconstructed Rp is nearly 3.3m. The Dist,Length/Width and Ratio are dependent highly on the Rp. The Figure 4 shows the one-dimensional distributions of Dist ,Ratio and Length/Width that after Rp correction.

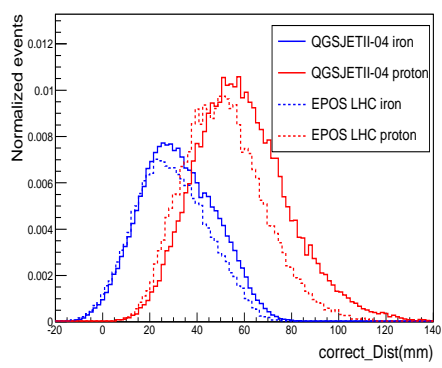

(a)

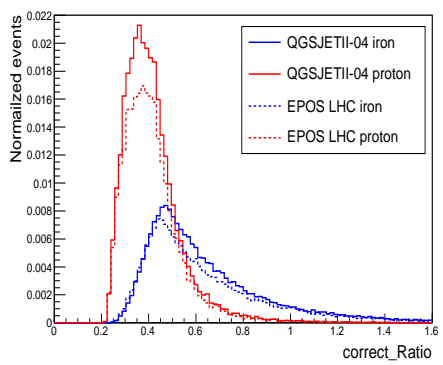

(b)

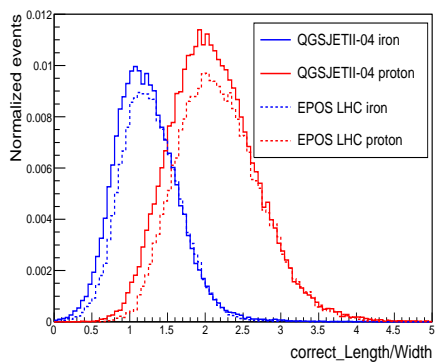

(c)

Figure 4: (a) One-dimensional distribution of Dist . (b) One-dimensional distribution of Ratio. (c) Onedimensional distribution of Length/Width. correct_Dist indicates the Dist after Rp correction ,correct_Ratio indicates the Ratio after Rp correction, correct_Length/Width indicates the length to width ratio after Rp correction .

After a"selecting cut" of raw image,the shower energy is reconstructed from the Hillas parameters of Size recorded by the WFCTA [8].The difference between the mean energy of proton and iron showers for the different models QGSJETII-04 and EPOS LHC are shown in Figure 5, the energy resolution is nearly about $25 \%$.

The discrimination of iron events from the hybrid events (iron and proton) is following the criterion that the purity of selection must larger than 0.90 . In this paper, the energy bin width is 


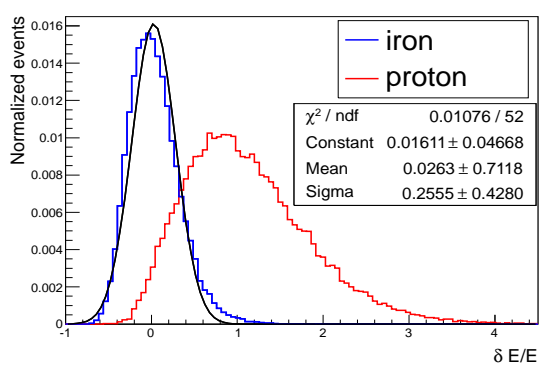

(a)

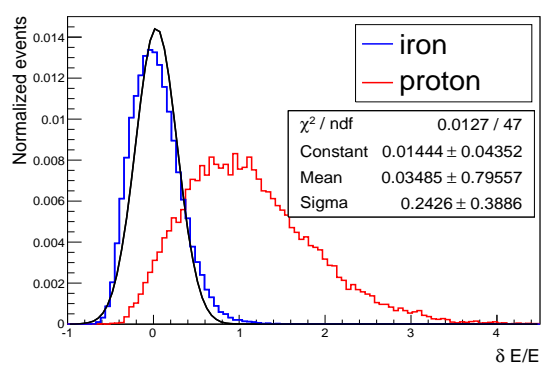

(b)

Figure 5: (a) The energy resolution of QGSJETII-04 .(b) The energy resolution of EPOS LHC . The dark lines indicates distributions are fitted with a Gaussian function.

chosen to be 0.12 in $\log _{10}(1 / \mathrm{GeV})$. The simulation process of composition discrimination divided into two steps. The first step use parameters of Dist and Length/Width for discrimination of iron events, the second step is use parameters of Dist, Length/Width and Ratio for discrimination of iron events. Selection efficiency is reported in the Figure 6(a). The WFCTA can only be operated in the clear moon-less nights, and the effective duty cycle is about $15 \%$. After one years' measurements,the number primary events and selected events by the difference steps in each energy bins are shown in Figure 6(b) and 6(c). The trigger efficiency increase with the energy, reaching the maximum 0.85 at energy $500 \mathrm{TeV}$.

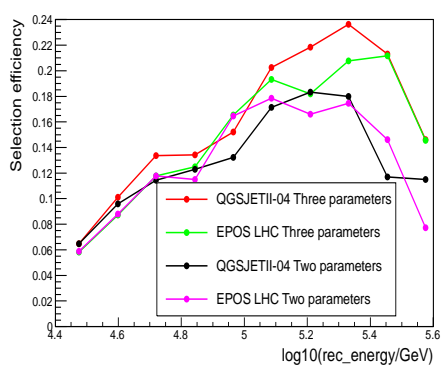

(a)

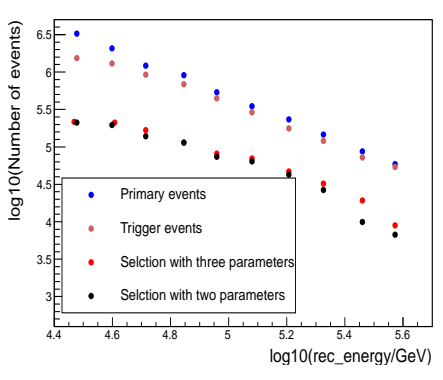

(b)

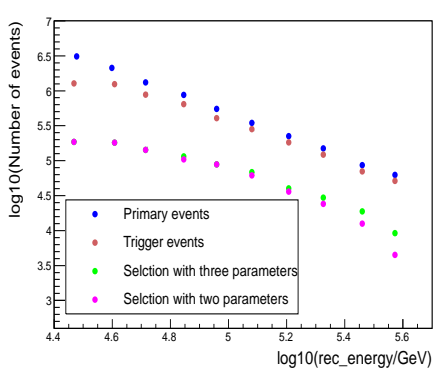

(c)

Figure 6: (a) The relationship between selection efficiency and reconstruction energy. (b) The relationship between the number of events and reconstruction energy for the model of QGSJETII-04. (c) The relationship between the number of events and reconstruction energy for the model of EPOS LHC.

\section{Summary and Discussion}

In this paper,under the criterion that purity is larger than 0.90 ,we add the new parameter of Ratio into the Hillas parameters(Length/Width and Dist). The rise of selection efficiency increase firstly and then decrease with energy for the model of QGSJETII-04, reaching the maximum values $83 \%$.However, the rise of selection efficiency increase with the energy for model of EPOS LHC ,reaching the maximum values $88 \%$,slightly larger than the rise of QGSJETII-04. The increase number of events of selection for add Ratio into the parameters of Dist and Length/Width is $9.4 \times 10^{4}(3.7 \%)$ for the model of QGSJETII-04. The increase events of selection is $2.8 \times 10^{4}(3.2 \%)$ 
for the model of EPOS LHC, slightly smaller than the rise for the QGSJETII-04. The independence of selection by three parameters for the model QGSJETII-04 is slightly larger than selection for the EPOS LHC.From the result in our simulation, we can conclude that the parameter of Ratio is defined via the characteristic of DC-light, to improve effectively of the resolution of reconstructed heavy nuclei spectrum.

Future improvements of the DC-light technique could extend the energy range of the measurement to energy of $1 \mathrm{PeV}$. Besides larger statistics, this extension requires improved separation power of the DC-light from the EAS-light, the reason for this is DC-light remains constant above a certain energy while the EAS-light yield increases approximately linearly with energy, as shown in Figure 1.Improved separation power can be achieved using the high resolution pixel array in the telescope camera .The reason is DC-light is emitted with a narrow Cherenkov angle $\left(0.1^{\circ} \sim 0.3^{\circ}\right)$, and high resolution pixel array improved the selection efficiency for the heavy nuclei , the fact testified experimentally by the telescope of HESS . Additionally separation power is also be achieved by time structure of the DC-light. Compare to the EAS-light,DC-light arrives with a typical delay of $4 \mathrm{~ns}$, this fact could not be exploited in the analysis via WFCTA, because the standard integration window of WFCTA is 20ns. However, in the planning Cherenkov telescopes, which consistently store pulse timing information, may take advantage of this characteristic [9] .

Acknowledgement: This work is supported by the National Natural Science Foundation (NSFC) of China under contacts No. 11635011 and No.11505190

\section{References}

[1] D.B.Kieda,S.P.Swordy ,and S.P.Wakely, Astroparticle Physics 15(2001) 287-303.

[2] Drury L C .An introduction to the theory of diffusive shock acceleration of energetic particles in tenuous plasmas .Rep Prog Phys,46:973-1027.

[3] T. Antoni et al., Astropart. Phys. 24, 1 (2005);

[4] J. Clem et al, Astropart. Phys. 16, 387 (2002).

[5] A. M. Hillas, astro-ph/0607109.

[6] http://www-ik.fzk.de/corsika/.

[7] J. R.Hördanel, Astropart. Phys. 19, 193 (2003).

[8] B. Bartoli et al, Chin. Phys. C 38, 045001 (2014).

[9] J. Holder et al., Astropart. Phys. 25, 391 (2006). 